\title{
Dark Matter in the Galactic Disk
}

\author{
John N. Bahcall \\ Institute for Advanced Study \\ Princeton, New Jersey 08540
}

\begin{abstract}
The Poisson and Vlasov equations are solved self-consistently for realistic Galaxy models which include multiple disk components, a Population II spheroid, and an unseen massive halo. The total amount of matter in the vicinity of the Sun is determined by comparing the observed distributions of tracer stars, samples of $\mathrm{F}$ dwarfs and of $\mathrm{K}$ giants, with the predictions of the Galaxy models. Results are obtained for a number of different assumed distributions of the unseen disk mass. The major uncertainties, observational and theoretical, are estimated. For all the observed samples, typical models imply that about half of the mass in the solar vicinity must be in the form of unobserved matter. The volume density of unobserved material near the Sun is about $0.1 M_{\odot} p c^{-3}$; the corresponding column density is about $30 M_{\odot} p c^{-2}$. This so far unseen material must be in a disk with an exponential scale height of less than $0.7 \mathrm{kpc}$. If the unseen material is in the form of stars with masses less than $0.1 M_{\odot}$, then the nearest such object is about $1 \mathrm{pc}$ away and has a proper motion of more than 1 arcsecond per year.
\end{abstract}

\section{Introduction}

The main results that I wish to convince you of are (Bahcall 1984a,b):

$$
0.5 \leq \frac{\rho_{\text {unobserved }}(0)}{\rho_{\text {observed }}(0)} \leq 1.5
$$

and

$$
z_{\text {scaleheight }} \leq 0.7 k p c
$$

The first equation says that the amount of unobserved material in the vicinity of the Sun is between 0.5 and 1.5 times the already observed material. The second equation says that the exponential scale height of the unobserved material, if it is a single population, must not exceed $0.7 \mathrm{kpc}$. Thus about half of the matter in the vicinity of the Sun is in the form of unseen disk material which has a scale height of less than $0.7 \mathrm{kpc}$. The unseen material that is inferred from galaxy rotation curves at large galactocentric distances and from applying the virial theorem to groups and clusters of galaxies may not be the same as the unobserved disk matter. 
As we have just heard from Sandy Faber and as Mike Turner will tell us in more detail, the unobserved material at large galactic radii and in clusters of galaxies is often discussed by particle physicists in terms of dissipationless particles (various 'inos'), while the unseen disk material is presumably dissipational. It is possible that the unseen material in the disk consists of stars or planets that are not massive enough to burn hydrogen and hence are of too low a luminosity to have been detected by searches carried out so far.

If you are willing to take the results in equations (1) and (2) on faith, you can doze through the rest of the talk without missing very much.

Before we get down to the justification of the main results, I want to remind you of a bit of the history of this subject because this perspective may have a special significance for the participants in this symposium. Oort's $(1932,1960)$ early studies of the total amount of matter in the solar vicinity led to what may have been the first astronomical suggestion of a large "missing mass." Nevertheless, most of the explanations for missing matter that will be discussed at this conference do not account for the unseen matter near the Sun.

\section{The Method}

The method of weighing the matter in the local neighborhood that I have used, and which Oort pioneered, can be summarized as follows. A detailed model of the observed matter (in stars, gas, and clouds) is constructed from all the available observations. In addition, the density distribution and velocity dispersion of a set of tracer stars perpendicular to the galactic plane is taken from published measurements. Theoretical models are then computed for the expected distribution of tracer stars in different gravitational potentials (mass distributions). The amount of matter that is actually present in the Galaxy is determined by comparing the observed and computed distributions.

The problem is similar to computing the distribution of an isothermal atmosphere (since for the tracer stars of interest the velocity dispersion changes much more slowly with height above the plane than does the density). Clearly, the more matter there is close to the plane. the more quickly will the density fall off with height above the plane.

The availability of modern computers has made possible important improvements in the theoretical analysis of this problem at the same time that better observational samples of tracer stars have been obtained. I have taken advantage of these developments to sharpen the determinations of the total amount of matter in the solar vicinity, using more realistic Galaxy models and more accurate theoretical solutions. I have solved numerically the combined Poisson and Vlasov equations for the gravitational potential of Galaxy models consisting of realistically large numbers of individual isothermal disk components in the presence of a massive unseen halo. Most previous calculations were carried out without requiring self-consistency between the Poisson and Vlasov equations. For example, in Oort's work the equations were solved separately. In the solutions that I will discuss, the distribution functions that solve Vlasov's equation for the observed matter and the tracer stars also depend on the potential that appears in Poisson's equation and generate, through their associated densities, the mass densities in Poisson's equation. I have carried out the calculations with different assumptions about the unseen matter and have compared the results with the observed number densities of $\mathrm{F}$ dwarfs and $\mathrm{K}$ giants versus height above the plane, assuming that the $\mathrm{F}$ dwarfs and $\mathrm{K}$ giants are reasonably faithful tracers of the total gravitational potential. Because the solutions are obtained with the aid of a computer, I can make more 
quantitative estimates of the errors by varying all of the parameters and by trying many different models.

Incidentally, the work of Oort and other previous investigators referred only to the equivalent of equation (1) above. The derivation of equation (2) requires combining the studies of the motion perpendicular to the plane with knowledge of the Galaxy rotation curve.

\section{The Input Data}

Table 1 summarizes the relative amounts of the observed mass components, and their velocity dispersions (i. e., temperatures) that were derived - using data from many sources - by Bahcall and Soneira (1980) and by Hill, Hilditch, and Barnes (1979), often referred to as the B\&S and the HHB Galaxy models. The models contain many observed disk components (typically 14) whose characteristics are determined by local measurements, a Population II spheroid inferred from faint star counts, different models for the unobserved disk components, and an unseen massive halo whose normalization is fixed by the solar rotation velocity. The mass fractions are defined in terms of the total observed mass density (in stars, gas, and dust), i. e.,

$$
A_{i}=\frac{\rho_{i}(0)}{\rho_{o b s}(0)} .
$$

I use the difference between the results obtained with the B\&S and the HHB Galaxy models as one measure of the uncertainty. The two models are similar, since the luminosity function of the disk stars is reasonably well determined (see Wielen 1974) over much of its range. The B\&S and HHD models mainly differ in the mass density assigned to white dwarfs and to the interstellar matter. In both cases, I have made use of more recent determinations. For example, fewer white dwarfs are observed at faint absolute magnitudes than had been expected on the basis of earlier theoretical estimates. I have used in the B\&S model the observed number density [ Green (1980), Liebert, Dahn, Gresham, and Strittmatter (1979)] down to $M_{V}=17.2$ and a white dwarf mass of $0.6 M_{\odot}$. I have also adopted the value for interstellar matter density that has been estimated by Spitzer (1978), which is consistent with the recent value inferred by Sanders, Solomon, and Scoville (1984). This value is rather larger than the interstellar matter density that was used by HHB.

Previous theoretical studies of the total amount of matter in the vicinity of the Sun have been limited mainly to simplified Galaxy models with one or, at most, a few disk components and no spherical component. The previous solutions were also limited either by what was tractable analytically or by assuming a numerical form for the total matter density that was independent of the potential. Since I have access to a VAX computer, I have calculated numerical models with many different sets of input data and a several assumptions about how the unseen material is distributed. I estimate the major uncertainties in the determination of the distribution of unseen matter by comparing an extensive collection of theoretical models with the available data.

\section{The Simplest Model for the Unseen Material}

Since we haven't yet observed the unseen material, we don't know how it is distributed. Therefore we have to try different models for the unseen material to see how the results depend upon our assumptions. 
Table 1

The Galaxy Model for Observed Components ${ }^{a}$

Component

(1)

$\begin{array}{lll}\text { B \& S Mass } & <v_{z}^{2}>^{1 / 2} & \text { HHB Mass } \\ \text { Fraction }\left(A_{i}\right) & & \text { Fraction }\left(A_{i}\right) \\ \left(M_{\odot} p c^{-3}\right) & \left(k m s^{-1}\right) & \left(M_{\odot} p c^{-3}\right)\end{array}$

(2)

(3)

(4)

Main Sequence Stars:

\begin{tabular}{llll}
$M_{V}<2.5 \mathrm{mag}$ & 0.021 & 4 & 0.038 \\
$2.5 \mathrm{mag} \leq M_{V} \leq 3.2 \mathrm{mag}$ & 0.015 & 8 & 0.019 \\
$3.2 \mathrm{mag} \leq M_{V} \leq 4.2 \mathrm{mag}$ & 0.031 & 11 & 0.033 \\
$4.2 \mathrm{mag} \leq M_{V} \leq 5.1 \mathrm{mag}$ & 0.035 & 21 & 0.034 \\
$5.1 \mathrm{mag} \leq M_{V} \leq 5.7 \mathrm{mag}$ & 0.025 & 20 & 0.023 \\
$5.7 \mathrm{mag} \leq M_{V} \leq 6.8 \mathrm{mag}$ & 0.037 & 17 & 0.036 \\
& 0.0358 & 8 & \\
& 0.0626 & 13 & \\
$M_{V} \leq 6.8 \mathrm{mag}$ & 0.0536 & 15 & 0.0262 \\
& 0.0626 & 20 & \\
Subgiants and Giants & 0.0834 & 24 & \\
White dwarfs & 0.016 & 20 & \\
Atomic H and He & 0.052 & 21 & 0.185 \\
& & & 0.287 \\
Molecular H and dust & 0.469 & 4 & \\
Spheroid & & & \\
Total & 0.001 & 100 & 0.083 \\
\hline & 0.0958 & $\ldots$ & \\
& & & \\
& & &
\end{tabular}

${ }^{a}$ Disk luminosity functions and velocity dispersions from Wielen (1974). 

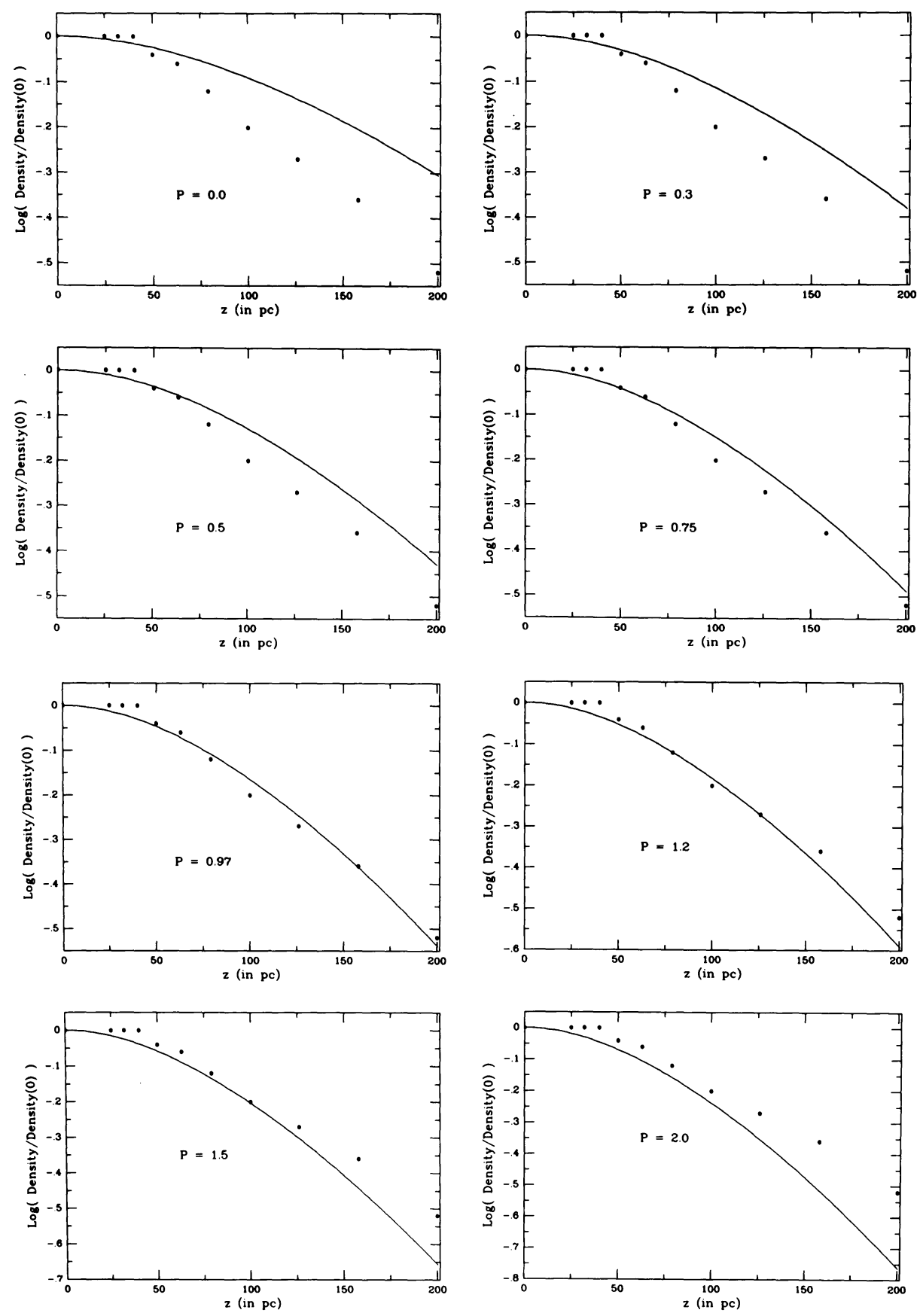

Figure 1. Comparison of measured versus computed number densities of $F$ stars. The measured densities are taken from the work of Hill et al. (1979). The mass in unobserved material is assumed to be proportional to the mass in observed material, stellar and interstellar, with proportionality constant P. 


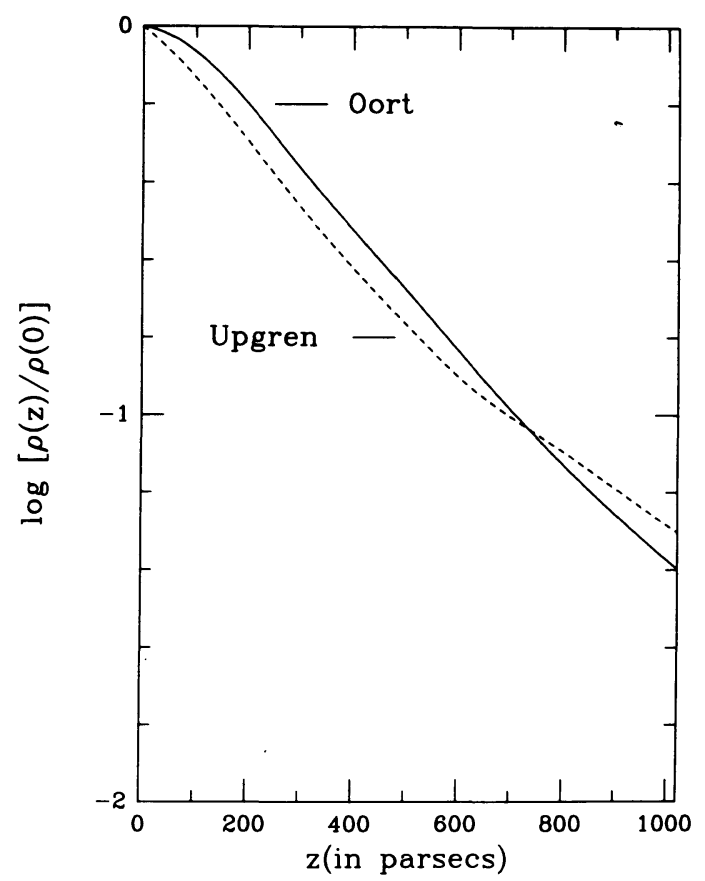

Figure 2. The comparison of the Oort (1960) and Upgren (1962) density distribution using the average visual magnitude and absorption adopted in Bahcall (1984b).

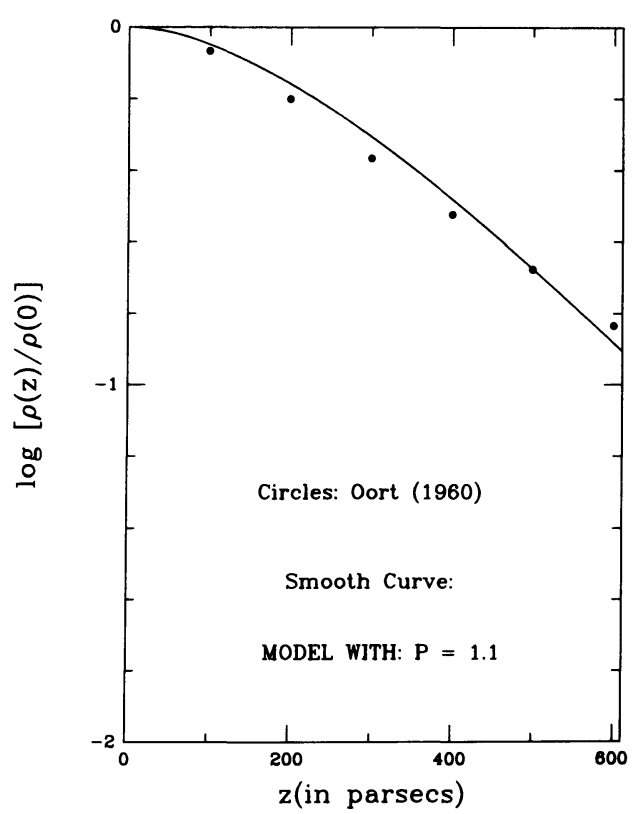

Figure 3. The best-fit model for the Oort K-giant data using the simple scale model defined in section III of this paper. 
There is one model which is uniquely simple and is characterized by only one parameter, the overall scale factor, $\mathrm{P}$, between observed and unobserved material. In this illustrative model, the unobserved mass density in every component, $i$, is proportional to the observed mass density in the same component,

$$
(\text { Observed })_{i} \equiv P \times A_{i}
$$

and the unobserved and observed velocity dispersions for the $i^{\text {th }}$ component are equal. Of course, this is only one of the many different models that have been explored.

Figure 1 is a chi-by-eye illustration of why one needs missing matter in the disk. I compare in this figure the measured star densities of Hill, Hilditch, and Barnes (1979) with a sequence of models computed assuming that the scale factor $\mathrm{P}=0.0,0.3,0.5,0.75,0.97,1.2,1.5$, and 2.0. You can judge for yourself the improvement in the agreement between model and observation as the amount of material is increased from no unobserved material $(P=0.0)$, through the best fit $(P$ $=0.97)$, to a worsening of the fit at large ratios of unobserved to observed matter (up to an unacceptable $\mathrm{P}=2.0$ ). For small values of $\mathrm{P}$, the observed distribution of $F$ stars falls off more rapidly than does the calculated distribution. Therefore, we have to add additional unseen matter to pull down the calculated curve. A formal statistical treatment (Bahcall 1984a) of the fit gives for this case $P=0.97 \pm 0.23$. Does that agree with your chi-by-eye assessment of the uncertainty? Incidentally, the flatness of the observed distribution within the first $40 \mathrm{pc}$ (the first three data points in Figure 1) is an artifact of the way that Hill et al. reduced their data and does not reflect any real observational constraint on the shape of the distribution at small heights above the plane.

Figure 2 shows the agreement between the number densities for the Oort(1960) and the Upgren(1962) samples of K giants. Figure 3 shows the best fit for the Oort data, again with the simple proportional model.

\section{Other Models and Equation (1)}

I have explored many possible models for the distribution of unobserved material. I have calculated, e. g., models in which the unobserved material has a small velocity dispersion (like the interstellar material), has a distribution like the older stars (e. g., like the white dwarfs or $\mathrm{K}$ giants), is distributed like all the observed stars (ignoring the interstellar material), or has the maximum scale height consistent with the Galaxy rotation curve.

Table 2 gives the ratio of unobserved to observed mass density for twentyeight detailed models (see Bahcall $1984 \mathrm{~b}$ for a description of these models) that fit the observed distribution of $\mathrm{K}$ giants. The models represent numerical solutions of the combined Poisson-Vlasov equation for different input parameters, as well as for several assumptions about the distribution of the unobserved disk material. There are separate columns referring to the observed K-giant samples of Oort (1960) and to the Upgren (1962) $\mathrm{K}$ giant density distributions. For both the volume and the column density, the typical best-fit model has, for the Oort densities, about equal amounts of unobserved and observed material. For the Upgren densities, the typical best-fit model has about $40 \%$ more unobserved than observed matter. These averages are only illustrative since at most one of the models considered for the distribution of unseen matter can be correct. Similar results are obtained by comparing theoretical models to the observed sample of $\mathrm{F}$ dwarfs (Bahcall 1984a). 
TABLE 2

RATIO OF UNOBSERVED TO OBSERVED DISK MATERIAL

\begin{tabular}{|c|c|c|c|c|}
\hline \multirow[b]{2}{*}{$\begin{array}{l}\operatorname{Row}^{a} \\
\text { (1) }\end{array}$} & \multirow{2}{*}{$\begin{array}{l}\text { Oort Densities } \\
\frac{\rho_{\text {unobs }}(0)}{\rho_{\text {obs }}(0)} \\
(2)\end{array}$} & \multirow[b]{2}{*}{$\begin{array}{l}\frac{\sigma_{\text {unobs }}}{\sigma_{\text {obs }}} \\
(3)\end{array}$} & \multicolumn{2}{|c|}{ Upgren Densities } \\
\hline & & & $\begin{array}{l}\frac{\rho_{\text {unobs }}(0)}{\rho_{\text {obs }}(0)} \\
(4)\end{array}$ & $\begin{array}{l}\frac{\sigma_{\text {unobs }}}{\sigma_{\text {obs }}} \\
(5)\end{array}$ \\
\hline $1 \ldots \ldots \ldots \ldots \ldots$ & 1.1 & 1.1 & 1.5 & 1.6 \\
\hline $2 \ldots \ldots \ldots \ldots$ & 1.6 & 1.6 & 2.1 & 2.1 \\
\hline 3.................. & 0.6 & 0.6 & 1.0 & 1.0 \\
\hline $4 \ldots \ldots \ldots \ldots$ & 0.9 & 0.9 & 1.3 & 1.4 \\
\hline $5 \ldots \ldots \ldots \ldots \ldots$ & 1.3 & 1.3 & 1.8 & 1.8 \\
\hline $6 \ldots \ldots \ldots \ldots$ & 1.3 & 1.1 & 1.8 & 1.6 \\
\hline $7 \ldots \ldots \ldots \ldots . .$. & 0.6 & 1.2 & 0.8 & 1.6 \\
\hline $8 \ldots \ldots \ldots \ldots . .$. & 0.7 & 0.7 & 1.0 & 1.0 \\
\hline $9 \ldots \ldots \ldots \ldots \ldots$ & 0.4 & 0.7 & 0.5 & 1.0 \\
\hline $10 \ldots \ldots \ldots \ldots$ & 2.4 & 0.5 & 2.6 & 0.5 \\
\hline $11 \ldots \ldots \ldots \ldots$ & 1.5 & 0.3 & 2.2 & 0.5 \\
\hline $12 \ldots \ldots \ldots \ldots$ & 0.6 & 2.5 & 0.7 & 3.2 \\
\hline $13 \ldots \ldots \ldots \ldots$ & 1.1 & 1.1 & 1.5 & 1.6 \\
\hline $14 \ldots \ldots \ldots \ldots$ & 1.5 & 1.5 & 2.0 & 2.0 \\
\hline Average.... & 1.1 & 1.1 & 1.5 & 1.5 \\
\hline
\end{tabular}

${ }^{a}$ Disk luminosity functions and velocity dispersions from Wielen (1974).

I conclude that a typical best-fit model implies that about half of the disk material at the solar position has not yet been observed. This conclusion, which is sunmmarized in equation (1), is in qualitative agreement with the previous major studies (see e. g., Oort 1932, 1960, Hill 1960, Woolley and Stewart 1967, Lacarrieu 1971, and Hill, Hilditch, and Barnes 1979), although I find a larger ratio of unobserved to observed matter than in some of the earlier analyses. The present investigation establishes more firmly and specifically the existence of unobserved disk material. The added confidence in the results arises because: 1) more realistic Galaxy models are used; 2) the Poisson and Vlasov equations are solved self-consistently; 3) improved (and more homogeneous) observational data are utilized; and 4) many theoretical models are compared with the observations in order to estimate the uncertainties. 


\section{But...}

I do not want to sound too satisfied, however. There is no modern data sample of $\mathrm{K}$ giants; the samples that I have been forced to use are a quarter of a century old! The stars are very bright (apparent magnitudes less than 10) so that it would be very easy to get a much improved sample with modern techniques, using spectroscopic observations to assure that the population was homogeneous with height above the plane. The velocity dispersions of both the $\mathrm{K}$ giants and the $\mathrm{F}$ dwarfs could be improved with modern radial velocity techniques. The absolute magnitude of the tracer stars should be redetermined using Hipparcos as well as the soon-to-bepublished Yale parallax catalogue.

The largest identifiable source of uncertainty in the Oort limit is the unknown form of the distribution of unseen matter (see the last row of Table 9 of Bahcall $1984 \mathrm{~b}$ ). In the future, it should be possible to constrain sharply the distribution of unseen matter by requiring consistency with observations of several carefully selected samples of tracer stars with different scale heights.

\section{The Rotation Curve and Equation (2)}

The unseen material must be mostly in a disk form, i.e., be dissipational. If all of the material were in a relatively round halo, then the rotation velocity at the solar position would have to be as large as $500 \mathrm{~km} \mathrm{~s}^{-1}$. For a given local volume density of unseen mass, the total amount of mass required in a round halo is larger than the amount of mass needed in a disk by about the ratio of the galactocentric distance of the Sun to the disk scale height, i.e., by more than an order of magnitude. The largest scale height of the unseen disk material that is consistent with the solar rotation velocity is $0.7 \mathrm{kpc}$ (see row 12 of Tables 5 and 6 of Bahcall 1984b). I determined this value by making a succession of models in which the unseen material had a progressively larger vertical velocity dispersion. For each model I required that the predicted distributions of tracer stars fit the observations of $F$ dwarfs and $\mathrm{K}$ giants and also be consistent with the observed $(220 \mathrm{~km} / \mathrm{s})$ rotation velocity at the solar galactocentric position. The maximum allowed vertical velocity dispersion is $40 \mathrm{~km} / \mathrm{s}$.

\section{What is it?}

If the missing material is in the form of stars that are not massive enough to burn hydrogen $\left(M<0.1 M_{\odot}\right)$, then the nearest such brown dwarf is probably less than a parsec away and has a proper motion of more than an arcsec per year. Brown dwarfs of the required number density might be detected in future dedicated large area surveys for very red, high proper motion objects. If the unseen material has a typical mass like that of Jupiter, the nearest such object would be about $0.2 \mathrm{pc}$ from the Sun, moving with a proper motion of order 5 arcseconds per year. Such remarkable objects might be discoverable with IRAS.

Moti Milgrom will describe at this conference the work he has done in constructing a theory of modified dynamics to account for missing matter (see, for example, Milgrom 1983 and references in the talk on this subject in the present volume). The modification that Milgrom has proposed implies that there should be missing matter in the z-direction. as well as in the plane of the Galaxy, and that this motion should be described by the simple scale model discussed in section IV of the present talk. In fact, the modified dynamics model predicts that $P \sim 1$, in agreement with the results summarized in equation (1) and in Table 2.

It may be that this agreement is just a coincidence. On the other hand, the value of $P$ could easily have been 0.1 or 10.0. I think we should be alert to the 
possible significance of the fact that the order of magnitude is the same for the "missing mass" inferred locally from the z-motion and globally from arguments about galactic halos. In most conventional models there must be two different explanations for the "missing mass". Perhaps there is a deep connection between the disk and the halo missing mass and they are both manifestations of the same phenomenon (which of course may have nothing to do with modified dynamics).

\section{The Harmonic Approximation}

Professor Einasto will report at this conference on the important investigations of Professor G. G. Kuzmin on the problem of determining the total amount of matter at the solar vicinity. The references to this work are mainly in the Russian literature and are contained in Professor Einasto's report. The Soviet work is not as well known in the West as it should be and therefore I will comment on the approximations that are involved.

The basic assumption that is made by Professor Kuzmin and his collaborators is that the gravitational potential is quadratic in height above the galactic plane, i. e.,

$$
\phi(z) \approx 2 \pi G \rho_{\text {total }}(0) z^{2}
$$

and therefore that the motion of the tracer stars is harmonic. The fractional error in the potential that is caused by this approximation is

$$
\frac{\Delta \phi}{\phi} \approx-\pi G \rho_{\text {total }}(0) z^{2} / 3 \sigma_{\text {total }}^{2}
$$

where $\sigma_{\text {total }}$ is a characteristic velocity dispersion for all of the matter (observed and unobserved). Suppose that we want to measure the total matter density using a set of tracer stars with a known velocity dispersion, $\sigma_{\text {tracer }}$. We need to have an accurate solution of Poisson's equation for at least two exponential scale heights of the tracer stars, i. e., we must have a solution that is valid for $z \leq z_{\text {tracer }}$, where:

$$
z_{\text {tracer }}^{2}=\sigma_{\text {tracer }}^{2} / \pi G \rho_{\text {total }}(0) .
$$

Let $\epsilon$ be the maximum allowed fractional error in the potential and insert equation (7) into equation (6). Then the velocity dispersion of the tracer stars must satisfy

$$
\sigma_{\text {tracer }} \leq(\epsilon / 0.1)\left(\frac{\sigma_{\text {total }}}{10 \mathrm{~km} / \mathrm{s}}\right) 5 \mathrm{~km} / \mathrm{s} .
$$

In addition, the sample of tracer stars must be relaxed and homegeneous. I do not know of any sample of observed stars that satisfies simultaneously equation (8) and the other requirements.

This work was supported in part by the National Science Foundation grants PHY-8217352 and by NAS8-32902.

\section{References}

Bahcall, J. N. 1984a, Ap. J. 276, 169.

Bahcall, J. N. 1984b, Ap. J. , $287,926$.

Bahcall, J. N. and Soneira, R. M. 1980, Ap. J. Suppl. 44, 73.

Green, R. F. 1980, Ap. J. 238, 685. 
Hill, E. R. 1960, Bull. Astr. Inst. Netherlands 15, .

Hill, G., Hilditch, R.W., and Barnes, J.V. 1979, M.N.R.A.S. 186, 813.

Lacarrieu, C.T. 1971, Astron. and Astrophys. 14, 95.

Liebert, J., Dahn, C. C., Gresham, M., and Strittmatter, P. A. 1979, Ap. J., 233, 226.

Milgrom, M. A. 1983, Ap. J. 270, 371.

Oort, J. H. 1932, Bull. Astr. Inst. Netherlands 6, 249.

Oort, J. H. 1960, Bull. Astr. Inst. Netherlands 15, 45.

Sanders, D. B., Solomon, P. M. and Scoville, N. Z. 1984, Ap. J. 276, 182.

Spitzer, L. 1978, Physical Processes in the Interstellar Medium

(New York: Wiley).

Upgren, A. R. 1962, A. J. 67, 37.

Wielen, R. 1974, Highlights of Astronomy, Vol. 3, 395, ed. Contopoulos, G., (Dordrecht: D. Reidel).

Woolley, R. and Stewart, J. M. 1967, M.N.R.A.S. 136, 329. 


\section{DISCUSSION}

LAKE: If you make the mass of the dark objects large enough, is there any chance that bound triples can masquerade as binaries? Have you actually done the dynamics of these things, and do you find reasonable masses for the stars?

J. BAHCALL: In many of the cases the stars in binaries with $0.1 \mathrm{pc}$ separations have identical radial velocities that are stable to better than $0.5 \mathrm{~km} \mathrm{~s}^{-1}$. These measurements are by D. Latham.

LAKE: But do you know enough about them to calculate a consistent mass function? Is there a chance that they could be bound triples with something of say $10 \mathrm{M}_{\odot}$ ?

J. BAHCALL: All that is required for my argument is that they exist, because they are disrupted so easily. There is no evidence for binary motion. They are so fragile that you can't put anything else in them.

OSTRIKER: You've convinced us that there is difficult-to-observe matter locally and that the amount is not negligible. But before we can speculate on what it might be, it would be useful for you to remind us what you've included in the observed matter, so that we don't try to add that in. For example, how many faint companions and how many white dwarfs of what luminosity are already in your observed sample?

J. BAHCALL: I integrated the Green-Liebert luminosity function for white dwarfs as far as it goes and used a mass of $0.6 \mathrm{M}_{\odot}$ for a typical white dwarf. For the interstellar medium, I include $\mathrm{HI}, \mathrm{HII}, \mathrm{He}, \mathrm{He}^{+}$ and $\mathrm{H}_{2}$ (found from $\mathrm{CO}$ measurements using Solomon's conversion factor Spitzer has a very similar estimate based on the reddening of nearby stars). I think that here I've erred by putting in too much - there's nothing like that amount within 100 or 200 pc of the Sun; we are in a hole. With respect to main-sequence dwarf stars, I integrated the Wielen luminosity function broken down into 11 components and used the individual velocity dispersion for each component. I have separated the subgiants and giants from the main sequence stars. The spheroid contributes $0.001 \mathrm{M}_{\odot} \mathrm{pc}^{-3}$.

OSTRIKER: And what fraction of the mass is assumed to be in normally unobserved companions?

J. BAHCALL: That's $25 \%$ of the $0.044 \mathrm{M}_{\Theta} \mathrm{pc}^{-3}$. Different prescriptions change that number by $\pm 10 \%$. However, it makes a difference of on $1 y$ $\sim 0.002 \mathrm{M}_{\odot} \mathrm{pc}^{-3}$ in the final answer.

SANDERS: You said that you put in the observed white dwarfs, but you mentioned that you did not include the number of white dwarfs that you expect from stellar evolution. What would that number be? 
J. BAHCALL: Wiedemann's estimate is two or three times bigger than the number I quoted. This is more than has been observed, but it's not a terribly big contribution.

SCHWARZSCHILD: You mentioned that Larson has a possible picture for increasing the number of white dwarfs. Could Larson be called on?

LARSON: If you stick to conventional models of galactic evolution, the latest one by Beatrice Tinsley predicts an amount of mass in white dwarfs which is something like a third of the mass in other ordinary forms (lower main sequence stars, interstellar gas and so on). So in answer to Martin's question: If you are willing to increase this number by revising the model of galactic evolution, you only need a factor of 3 change to get the amount of mass in remnants to be equal to the amount of ordinary matter in other forms. I have a poster paper which suggests a way of doing this. It uses a model in which the initial mass function is not a Salpeter power law, but is bimodal. In that kind of model you can quite easily get enough mass in remnants of 2,3 or $4 \mathrm{M}_{\odot}$ stars to give you the factor of 3 increase.

I have another comment, as to whether the dark matter could be very low mass stars. I don't think there's any suggestion of this in the available data. It has been clear for a long time that there are few stars known that have $M<0.1 \mathrm{M}_{\odot}$. There may be very few out there. The latest discussions of the luminosity function of faint stars are two extensive preprints, one by Scalo and one by Poveda. They agree that there's a peak at around 0.2 or $0.5 \mathrm{M}_{\odot}$, followed by a fairly steep decline toward lower masses. This luminosity function implies a negligible mass in stars less massive than $-0.1 \mathrm{M}_{\odot}$. To have a lot of stars with masses less than $0.1 \mathrm{M}_{\odot}$, the luminosity function would have to be quite remarkable, with another peak at very faint luminosities.

J. BAHCALL: I think you've correctly summarized the general opinion. I am less confident of these conclusions than are some of my colleagues. Isn't a peak between 0.1 and $0.01 \mathrm{M}_{\odot}$ just as likely as one between 0.5 and $2 \mathrm{M}_{\odot}$ ? I guess the general answer to Martin's question is that Larson's paper makes it theoretically acceptable to consider bimodal distributions. For me, that was the main point of his paper, which I liked very much.

SCHMIDT: Your statement was that if the unseen mass is included in the Luyten luminosity function, the slope has at least to be between 0.01 and 0.05 or so?

J. BAHCALL: Yes. I know that this conflicts with your result and with a number of others. I'm not absolutely convinced, however.

FABER: A question for Larson. When you were describing conventional models and the amount of mass in remnants, was the star formation rate uniform or was it slightly declining as a function of time?

LARSON: Uniform. 
FABER: And is that still your feeling now?

LARSON: Such evidence as there is suggests that it has been uniform, but that evidence only applies to a restricted range of masses $\left(\sim 1 \mathrm{M}_{\odot}\right)$. Maybe the stars that formed at earlier times were more massive, a suggestion first developed seriously by Maarten Schmidt.

PACZYNSKI: I understand that all of your models assume for simplicity that the distribution of the ISM in the galactic plane is smooth. Yet there's this hole near the Sun, 100 pc across. Obviously, this doesn't affect the behavior of stars at $z$ distances of $600 \mathrm{pc}$; these stars average over a large area of the disk. However, when you are closer to the disk, the existence of the hole might be felt. How does it affect the slope of the decrease in stellar density with $z$ ?

J. BAHCALL: We11, as an example, I made an extreme model containing no ISM, and it was within the range of acceptable mode1s. One can also ask questions like: How massive would a molecular cloud at the edge of the hole have to be to have an effect? The answer is a very high mass, $\sim 10^{8} \mathrm{M}_{\odot}$.

WHITE: All of your models assume a Gaussian velocity distribution for each component and a velocity dispersion which is independent of height above the galactic plane. How well do the observational data support this independence for the $F$ stars and the $\mathrm{K}$ giants on which you rely most heavily? Specifically, are there sufficient stars nearby and at $z$ greater than one scale height to be sure that the dispersions of the two samples do not differ by more than $30 \%$ ?

J. BAHCALL: The $\mathrm{K}$ giant velocities that $\mathrm{I}$ have studied are consistent with a Gaussian distribution and a constant velocity dispersion to the accuracy of the measurements. This work is shown in Table 1 and Figure 1 of Ap. J., 287, 926 (1984). An essential and new element in this analysis is to use only normal-metallicity disk objects. The fractional change in the velocity dispersion was < $10 \%$ over the entire distance surveyed. The F-star data are less extensive but are also consistent with a constant velocity dispersion within the heights I have studied. For both samples, I have calculated the maximum effect allowed due to departures from isothermality, and found a change of only a few percent of the local matter density.

P. QUINN: Can you constrain the scale length of the dark matter in the disk? If not, could the scale length be very large and affect the shape of the rotation curve?

J. BAHCALL: I can't constrain the disk scale length from my arguments. When we found an upper limit of $0.65 \mathrm{kpc}$ for the scale height of the dark material, Soneira and I used a disk scale length of $3.5 \mathrm{kpc}$. If we use van der Kruit's larger value of $5.5 \mathrm{kpc}$, that 1imit rises to 0.7 kpc. 
TREMAINE: I guess I'm still a little confused about how much you have to stretch the conventional picture to get rid of the missing mass in the disk. I can easily imagine that you have an extra 0.01 or $0.02 \mathrm{M}_{\odot}$ $\mathrm{pc}^{-3}$ in molecular gas and about the same amount in unobserved white dwarfs. That would bring the observed density of $0.11 \mathrm{M}_{\odot} \mathrm{pc}^{-3}$ up to $0.14 \mathrm{M}_{\odot} \mathrm{pc}^{-3}$. This seems to be getting within a standard deviation or two of the Oort limit.

J. BAHCALL: You have to get the observed mass up to $0.2 \mathrm{M}_{\odot} \mathrm{pc}^{-3}$.

TREMAINE: OK. Let me phrase it another way. How embarassed would you be if all this went away?

J. BAHCALL: It won't go away. I'm confident of that.

MATHIEU: A comment on open star clusters. To date the dynamics of three clusters varying in age from a few times $10^{7}$ to a few times $10^{9}$ years have been studied. Two of these, M1 1 and M67, are discussed in a poster paper. In all three cases, the agreement of the observed velocity dispersion with that predicted from the stellar spatial distribution is good. There is no evidence for dark matter in these systems. However, due to mass segregation, the observed velocity dispersions are not sensitive to objects with masses less than $1 \mathrm{M}_{\odot}$. So if we consider the possibility that the disk dark matter consists of objects formed in typical star-forming regions and that the open clusters are a microcosm of the disk population, then these results suggest that the dark matter is in objects with masses less than $\sim 1$ $\mathrm{M}_{\odot} \cdot$

J. BAHCALL: That is an important comment. It is consistent with the argument based on the existence of wide binaries.

SHUTER: I have the impression, which could be confirmed by an analysis of the Bell Labs ${ }^{13} \mathrm{CO}$ Galactic Plane survey, that there is a missing mass problem of comparable magnitude in Giant Molecular Clouds. The mass estimated from their dynamics is a factor of 2-3 greater than that estimated from the CO column density. If this is the case, the unobserved material must have relatively small random velocity to be captured in molecular clouds. It must therefore have a small scale height, perhaps comparable to that of $\mathrm{CO}$ clouds in the solar neighborhood, which is $\sim 85$ pc.

J. BAHCALL: Very interesting. 